\title{
PROPOSTA DE SEQUÊNCIA DIDÁTICA PARA \\ DISCIPLINA DE QUÍMICA GERAL EXPLORANDO O USO DE TECNOLOGIAS DIGITAIS
}

Julliane D. Yoneda ${ }^{1}$

José Augusto Oliveira Huguenin ${ }^{1}$

\section{RESUMO}

Este artigo apresenta um relato de experiência de uma proposta de sequência didática integrada envolvendo as disciplinas de Química Geral e Introdução à Computação, ambas para alunos do primeiro semestre de cursos de graduação em Química. As atividades foram planejadas com base em aspectos básicos da Teoria de Aprendizagem Significativa de Ausubel. O uso de análise de dados e a confecção de gráficos em ambientes computacionais, além da aplicação de computação simbólica, mostraram ser ferramentas interessantes no ensino de conceitos de Química. A proposta foi aplicada, e, com isso, observou-se um maior engajamento dos alunos no processo ensino-aprendizagem e um melhor aproveitamento dos estudantes nas avaliações. A sequência didática mostrou ter potencial para aplicação em outras disciplinas de Ciências Exatas do ensino superior.

Palavras-chave: Tecnologia de Informação e Comunicação. Ensino de Química. Aprendizagem Significativa.

\footnotetext{
${ }^{1}$ Instituto de Ciências Exatas, Universidade Federal Fluminense (UFF), Volta Redonda, RJ, Brasil.
} 


\title{
PROPOSAL OF A DIDACTIC SEQUENCE FOR GENERAL CHEMISTRY DISCIPLINE BY USING DIGITAL TECHNOLOGIES
}

Julliane D. Yoneda

José Augusto Oliveira Huguenin

\begin{abstract}
This article presents an experience report about a proposal of an integrated didactic sequence involving General Chemistry and Introduction to Computing disciplines, for first semester students of undergraduate courses in Chemistry. The activities were planned based on basic aspects of Ausubel's Meaningful Learning Theory. The use of data analysis, graphing in computational environments, and the application of symbolic computation have proved to be interesting tools in the teaching of Chemistry concepts. The proposal was tested and there was a greater engagement of students in the teaching-learning process and a better performance in their evaluations. The didactic sequence showed potential for application in other disciplines of high education Sciences.
\end{abstract}

Keywords: Digital Technologies. Teaching Chemistry. Meaningful Learning. 


\section{INTRODUÇÃO}

O ensino de forma geral, notadamente na área de Ciências, vive um momento crucial. A sala de aula, sobretudo no Ensino Superior, em cursos de graduação, pouco mudou nos últimos trinta anos, enquanto os alunos da chamada "geração digital" trazem consigo uma nova forma de interagir socialmente através de redes sociais virtuais, além de serem dotados de habilidades para lidar com aplicativos e equipamentos eletrônicos (PONTE, 2011). Assim, uma linguagem que tem potencial para ser mais efetiva na comunicação com os alunos é a linguagem tecnológica, ou seja, através do uso de Tecnologias de Informação e Comunicação (TICS). Podese denominar TIC um conjunto de sistemas computacionais (hardware e software) capazes de automatizar informações, simular processos. Em suma, o uso de recursos computacionais pode ser considerado uma TIC. Devido ao avanço dos dispositivos computacionais, podemos melhor denominar esses recursos como Tecnologias Digitais (TD). A aplicação de tais tecnologias no ensino tem sido muito discutida (PEIXOTO; ARAÚJO, 2012).

Nos últimos anos, trabalhos que discutem e investigam o uso de TD no ensino de Química têm crescido (SERRA, 2009). No campo da ludicidade, jogos digitais com enfoque educativo voltados ao ensino de Química têm sido produzidos (LIMA; MOITA, 2011; PAIVA; RAMOS, 2004). Tavares, Souza e Correia (2013) apresentam uma discussão sobre o uso de TD no ensino de Química, acompanhada de um levantamento de portais e sites que oferecem interatividade. Teruya et al. (2013) mostraram que muitos trabalhos apontam para a importância do uso de visualizações no ensino de Química, ensino que pode ser potencializado com o uso de novos aplicativos e programas de computador.

Para o ensino de Química em nível superior (na graduação), outro fator importante a ser considerado é o uso de TD para auxiliar em aspectos formais e matemáticos, sempre um fator considerado crítico em cursos de ciências básicas, como Química e Física. Através de integração numérica por meio do software SCILAB, leis de velocidade diferenciais de reações foram estudadas, mostrando o potencial didático do uso de computação (SANDES; AMBROSIO; ANGELUCCI, 2013). O uso de planilhas também foi explorado para o ensino da Tabela Periódica (PINHEIRO et al., 2014).

O uso de teorias de aprendizagem desponta como alternativa na construção de sequências de ensino na graduação em Química (MENDONÇA; LEITE; RODRIGUES, 2009), tomando como base a Teoria de Aprendizagem Significativa (TAS) (AUSUBEL, 1968). Leite (2015) mostra uma 
gama de aplicações de TDs associadas a diferentes teorias de aprendizagem, inclusive a TAS, na formação de professores de Química.

Bogisch e Alcantara (2002) mostraram que aulas expositivas dialogadas associadas a atividades práticas promovem bons resultados no processo ensino-aprendizagem de Química. Dessa forma, acredita-se que as aulas expositivas dialogadas, no contexto da TAS, podem promover a participação do aluno, demandando interação dos estudantes com seus conhecimentos prévios através de problematizações que iniciam as discussões.

O objetivo deste trabalho é apresentar o relato de experiência de aplicação de uma sequência didática desenvolvida por uma ação conjunta entre duas disciplinas introdutórias para ingressantes nos cursos de Bacharelado em Química Tecnológica e Licenciatura em Química, do Instituto de Ciências Exatas da Universidade Federal Fluminense: Química Geral (QG), do Departamento de Química, e Introdução à Computação (IC), do Departamento de Física. A primeira tem o caráter de formação básica do curso de Química, enquanto a segunda apresenta ferramentas computacionais úteis ao desenvolvimento acadêmico dos alunos. Os conteúdos das disciplinas foram articulados de forma a explorar o aprendizado de pacotes e aplicativos computacionais como auxílio na compreensão e na operacionalização de conceitos de Química.

\section{FUNDAMENTAÇÃO TEÓRICA: APRENDIZAGEM SIGNIFICATIVA}

A TAS, desenvolvida por David P. Ausubel (1968) encara o processo ensino-aprendizagem como transformação da estrutura cognitiva do aprendente. A teoria se desenvolveu com grande contribuição de Novak (AUSUBEL; NOVAK; HANESIAN, 1978) e do Prof. Marco Antônio Moreira (MOREIRA, 2011), um dos desenvolvedores da TAS e o seu maior divulgador no Brasil. Por esse motivo, a abordagem aqui apresentada toma como base a profícua produção de Moreira no tema. Fator central dessa teoria, o conhecimento prévio trazido pelo aluno é o que mais influencia no aprendizado do estudante. A interação do novo conhecimento com o que já se encontra ancorado na estrutura cognitiva é um caminho essencial para o aprendizado. Os conhecimentos "ancorados" são identificados como subsunçores (MOREIRA, 2012). Quando a aprendizagem ocorre, esses subsunçores são modificados na estrutura cognitiva, ou mesmo novos subsunçores são criados, e, dessa forma, há maior significado para o aprendente diante do tema de estudo. Progressivamente, o aluno 
passa a aprender cada vez mais em função dos subsunçores já construídos e a mediação pessoal (geralmente do professor) passa a ser uma negociação de significados, aceitos e não aceitos, no contexto de um determinado corpo de conhecimentos (MOREIRA, 2011, p. 29).

A aprendizagem significativa ocorre através de conhecimentos prévios e novos de forma não literal (o aprendiz dá significado ao conhecimento; cada indivíduo dará um significado próprio, de acordo com a sua estrutura cognitiva) e não arbitrária (o novo conhecimento não interage com um subsunçor qualquer). Basicamente, pode-se dizer que se trata do oposto da chamada aprendizagem mecânica, que é literal (não dá margem à negociação de significados) e apropriada ao modelo narrativo de ensino, muito utilizada no ensino de Ciências e aceita pela sociedade de forma geral (MOREIRA, 2011).

Ao se considerar que a estrutura cognitiva pode ser entendida como uma cadeia de subsunçores inter-relacionados de forma hierárquica, sua dinâmica é caracterizada por dois processos básicos: a diferenciação progressiva e a reconciliação integradora. Chama-se diferenciação progressiva o resultado da sucessiva utilização de um dado subsunçor, na qual este ganha, progressivamente, novos significados (MOREIRA, 2011). A dinâmica do uso dos subsunçores é fundamental para a aprendizagem pois

Através de sucessivas interações, um dado subsunçor vai, de forma progressiva, adquirindo novos significados, vai ficando mais rico, mais refinado, mais diferenciado, e mais capaz de servir de ancoradouro para novas aprendizagens significativas (MOREIRA, 2011, p. 20).

Por outro lado, a reconciliação integradora, que ocorre simultaneamente à diferenciação progressiva, é responsável por eliminar diferenças aparentes e integrar significados no processo de aprendizagem. É por meio destes dois processos dinâmicos e simultâneos que o indivíduo vai hierarquizar e superordenar antigos e novos conhecimentos em sua estrutura cognitiva (MOREIRA, 2011). Esses dois processos podem, então, servir de guia para a construção curricular ou o planejamento das disciplinas, identificando ideias mais gerais, o que Moreira (2011) chamou de conceitos estruturantes, de forma a iniciar o ensino de um dado conteúdo por seus aspectos gerais e, progressivamente, ir para aspectos mais específicos, ou seja, promover a diferenciação progressiva. Nessa abordagem,

Ao longo de todo curso de uma disciplina, por exemplo, os conteúdos gerais e específicos devem ser trabalhados em uma perspectiva 
de diferenciação e integração, de descer e subir, várias vezes, nas hierarquias conceituais (MOREIRA, 2011, p. 43).

Ainda de acordo com Moreira (2011):

No caso de um conteúdo científico, por exemplo, que esteja organizado dentro de duas ou três leis científicas, a abordagem desse conteúdo deveria começar com estas leis, mas do ponto de vista fenomenológico e conceitual. Progressivamente, as mesmas seriam exemplificadas e modeladas matematicamente, em níveis crescentes de complexidades, até alcançar-se o nível esperado no contexto da disciplina (MOREIRA, 2011, p. 44).

Sendo a aprendizagem significativa não arbitrária, uma dificuldade presente em muitos alunos é justamente fazer a interação do novo conhecimento com o subsunçor correspondente. Há também a possibilidade de o aluno não possuir o subsunçor adequado. Nesses casos, entram os chamados organizadores prévios, que podem ser considerados recursos instrucionais que visam suprir a carência de conhecimentos prévios em determinados assuntos. Moreira (2011) aponta que esses organizadores prévios podem ser "um enunciado, uma pergunta, uma situação problema, uma demonstração, um filme, uma leitura introdutória, uma simulação. Pode ser também uma aula que precede um conjunto de aulas" (MOREIRA, 2011, p. 30). Os organizadores prévios podem ser classificados em expositivos (quando o aprendiz não possui subsunçores e o tema não lhe é familiar) e comparativos (quando o objeto de estudo é familiar ao aprendiz). O segundo caso ajuda o aluno a dar significado ao novo conhecimento, mas também discriminá-lo de conhecimentos presentes em sua estrutura cognitiva que possam ser confundidos.

Em outras palavras, organizadores prévios podem ser usados para suprir a deficiência de subsunçores ou para mostrar a relacionalidade e a discriminabilidade entre novos conhecimentos e conhecimentos já existentes, ou seja, subsunçores. [...] organizadores prévios devem ajudar o aprendiz a perceber que novos conhecimentos estão relacionados a ideias apresentadas anteriormente, a subsunçores que existem em sua estrutura cognitiva prévia (MOREIRA, 2011, p. 30-31).

Moreira (2011) aponta duas condições básicas para que possa ocorrer a aprendizagem significativa: 1) o material ser potencialmente significativo, ou seja, que o material que apresenta o conteúdo (livros, filmes, artigos, aulas) seja relacionável com conhecimentos relevantes dos conteúdos que aborda de forma não arbitrária e não literal; e 2) o aluno 
deve ter uma predisposição ao aprendizado, isto é, uma predisposição a relacionar os novos conhecimentos aos conhecimentos prévios, o que pode não ser tão simples, pois tal predisposição vai além da mera motivação do aluno (MOREIRA, 2011).

Há fatores que podem facilitar a aprendizagem significativa, resumidos da seguinte forma: a) levar em conta o conhecimento prévio do aluno ou, na impossibilidade de se fazer isso na prática, buscar construir a apresentação dos conceitos de modo a facilitar a diferenciação progressiva e a reconciliação integradora, conforme discutido acima; b) usar organizadores prévios; c) utilizar a organização sequencial, ou seja, explorar as relações hierárquicas naturais existentes entre conceitos de um dado assunto; d) consolidar os conhecimentos prévios antes de iniciar o estudo de novos conhecimentos; e f) utilizar uma linguagem adequada para apresentação dos conteúdos de forma a facilitar a identificação de subsunçores e a correta significação do conceito (MOREIRA, 2011).

Evidentemente a breve discussão apresentada nesta seção não aborda toda amplitude e abrangência da TAS. Optou-se por mostrar aspectos básicos da teoria que inspirou e fundamentou a proposição da sequência didática apresentada neste trabalho.

\section{ESTRATÉGIAS PARA CONTRUÇÃO DA SEQUÊNCIA DIDÁTICA INTEGRADA}

A proposta de sequência didática integrada foi construída a partir de três pilares:

Interdisciplinaridade: é um aspecto valorizado nos documentos oficiais de educação, como os Parâmetros Curriculares Nacionais (PCN), para os quais é por meio dessa interdisciplinaridade que se estabelecem "[...] interconexões e passagens entre os conhecimentos através de relações de complementariedade, convergência ou divergência" (BRASIL, 2002, p. 36). Para Fazenda (1994), a interação de disciplinas para o ensino de conteúdos se dá mediante o que ela chamou de "parcerias" que promovem o diálogo entre diferentes formas de conhecimentos, sendo essas parcerias o fundamento da ação interdisciplinar no ensino. Assim, toma-se o conceito de parcerias (FAZENDA, 1994) entre as disciplinas de QG e IC de forma a se criar um ambiente de colaboração. Mais especificamente, utilizou-se a chamada "interdisciplinaridade auxiliar", classificação devida a Heinz Heckhausen, que ocorre com a associação de duas ou mais disciplinas (CARLOS, 2007). 
Aulas expositivas dialogadas: essas aulas rompem a tradição imperante em cursos de Ciências Exatas, que empregam em larga escala um modelo narrativo (MOREIRA, 2012) que impede a reflexão dos estudantes sobre os conceitos, contribuindo para uma aprendizagem mecânica também na universidade. Uma aula dialogada exige que o aluno se posicione ante situaçõesproblema e possa confrontar seus conhecimentos prévios sobre o assunto. Essa postura tira o conforto dos estudantes que só "copiam" ou, mais recentemente, fotografam o quadro/slide do professor. Essa abordagem permite, de forma natural, como será visto na próxima seção, o uso de ferramentas típicas da TAS - como a introdução de organizadores prévios - e, no processo dialógico, o "ir e vir" no conteúdo, de forma a facilitar a diferenciação progressiva e a reconciliação integradora.

Uso de TICs (aplicativos e softwares matemáticos): seguindo a definição de Moreira (2011) para "materiais potencialmente significativos", discutida na seção anterior, acredita-se que o uso de TICs pode facilitar a relação de conteúdos com conhecimentos prévios de forma não literal e não arbitrária. Em cursos superiores de Química, os conceitos precisam ser manipulados por relações matemáticas, no que o uso de aplicativos de operação matemática pode ajudar. Devido à imersão das novas gerações a ambientes digitais, tecnologias de informação tornaram-se linguagens apropriadas para discutir conceitos científicos. A facilidade na realização de cálculos permite aos alunos, por exemplo, a possibilidade de explorar parâmetros e visualizar melhor a dependência com variáveis importantes nos conteúdos estudados, enfim, potencializa a interação do aprendiz com os conceitos estudados.

\section{PROPOSTA DE SEQUÊNCIA DIDÁTICA INTEGRADA PARA CONTEÚDOS DE QUÍMICA GERAL}

Para elaboração de aulas ou sequências didáticas baseadas em TAS, vale frisar, deve-se levar em conta o conhecimento prévio do aluno. Moreira (2011) aponta que, na impossibilidade de levantar os conhecimentos prévios explicitamente antes da aula, deve-se utilizar os chamados conceitos estruturantes, abordando o conteúdo a partir de aspectos gerais e fenomenológicos, até chegar a aspectos mais específicos do conteúdo. Nessa abordagem é observada a importância do uso de organizadores prévios.

Apresenta-se a seguir uma célula mínima da sequência, para facilitar a compreensão dos passos e das estratégias para cada etapa. Pode-se resumir a sequência da seguinte forma: 


\section{Etapa 1: Encontro de QG - aula expositiva dialogada com problematizações}

O primeiro encontro se inicia com o uso de um organizador prévio. Em consonância com a estratégia de aulas expositivas dialogadas, esse organizador consistiu de perguntas iniciais. Para elaboração dessas perguntas, é necessário identificar os conhecimentos prévios mais relevantes para o conteúdo a ser ensinado e que, idealmente, o aluno deveria ter para formular as perguntas-organizador prévio.

Se houver folga no calendário, esse primeiro encontro poderia ser inteiramente devotado a discussões fenomenológicas do conteúdo, por exemplo, a partir de perguntas conceituais para diálogo entre os alunos com mediação do professor. Contudo, se o calendário for apertado para cumprimento da ementa da disciplina, recomenda-se a especificação do conteúdo já nesse momento, de forma a explorá-lo no Encontro de IC, que vem logo a seguir. Idealmente, deixa-se uma pergunta para ser respondida na aula de computação.

\section{Etapa 2: Encontro de IC - aula expositiva dialogada com o uso de recursos computacionais}

Nesta etapa são introduzidos softwares e aplicativos que serão utilizados. A introdução deles é realizada a partir da problematização/questão/pergunta deixada no primeiro Encontro de QG. Essa pergunta, ou semelhante, deve poder ser respondida com o uso de recursos computacionais. A retomada da situação em ambiente diferente (interdisciplinar) dá ao aluno a possibilidade de "ir e vir" no conceito, oportunizando de forma intensa a diferenciação progressiva e, também, possibilitando a reconciliação integradora.

Idealmente, as aulas com recursos computacionais devem ser feitas em laboratório didático de informática, onde os alunos poderão manipular os softwares e explorar a solução do problema. Na impossibilidade de aulas em laboratórios de informática, é possível projetar em sala de aula o uso dos aplicativos matemáticos, o que se assemelha a aulas demonstrativas. 


\section{Etapa 3: Encontro de QG - aula expositiva dialogada sobre o tema e sistematização do conteúdo}

Nesta parte da sequência, o problema já está colocado, o aluno já foi apresentado a conhecimentos novos no primeiro encontro, visando à resolução da questão problematizadora. Nesse ponto, já foram observadas, também, algumas ferramentas computacionais/ matemáticas. Esse é o momento de formalização e sistematização do conteúdo.

Fechando a sequência didática, esse terceiro encontro de QG vai sistematizar o conhecimento em aula expositiva dialogada, explorando a experiência nas aulas de IC. É feita uma discussão geral e integradora. Nesse momento os alunos terão a visão geral do conceito e da sua formalização de forma mais parecida com uma aula tradicional. Esse encontro é fundamental, pois oportunizará o preenchimento de lacunas e esclarecimento de possíveis confusões. Em outras palavras, permitirá que ocorra de forma mais intensa a reconciliação integradora. $\mathrm{Na}$ etapa 3 ocorre também a discussão de outras situações ou mesmo a resolução de exercícios.

Ao fim deste encontro, uma nova situação-problema pode ser colocada, iniciando-se a sequência para um novo tópico/conteúdo.

O tempo usado em cada etapa pode variar conforme a disponibilidade e a demanda de tempo do docente. Para conceitos mais complexos, com diferentes nuances, as etapas 1 e 2 podem se repetir antes da sistematização da etapa 3. Apesar de a sequência apresentada ter explorado o fato de haver uma disciplina de computação no mesmo período da disciplina QG, possibilitando a ação integrada, a proposta pode ser utilizada, também, apenas dentro da disciplina de QG.

A sequência didática integrada apresentada é bem geral, com etapas bem estabelecidas, e pode ser aplicada para diferentes conteúdos. Para seu uso, deve-se seguir um protocolo simples de preparação:

1. Definição de conteúdo: ao definir o conteúdo que será ensinado com a sequência proposta, deve-se estabelecer o que pode ser explorado computacionalmente, preparar as perguntas que serão os organizadores prévios e definir as situaçõesproblema que introduzirão o conteúdo. 
2. Definição de recursos computacionais: escolher, entre os recursos computacionais disponíveis, aqueles que melhor podem ser explorados para o conteúdo a ser ensinado.

Para exemplificar a aplicação da proposta, dois exemplos são apresentados na sequência.

\section{Definição de conteúdo}

Os temas de Cinética Química e Equilíbrio Químico foram selecionados para aplicação da abordagem proposta. O primeiro porque a construção de gráficos com auxílio do computador permite a visualização e a melhor compreensão de como avaliar a cinética de uma reação química, bem como determinar constantes de velocidade. O segundo porque em muitos problemas o aluno se depara com equações de terceiro grau e precisa fazer aproximações para conseguir resolvê-las.

\section{Definição de recursos computacionais}

Para os tópicos selecionados, são necessários recursos computacionais que sejam capazes de resolver equações de grau maior que dois (terceira e quarta ordem), por um lado, para estudo de problemas de Equilíbrio Químico e geração de gráficos e ajustes de funções, por outro lado, para os estudos de Cinética Química.

Para a solução de equações ou sistemas de equações, um recurso de grande poder e simplicidade é o uso de linguagem simbólica ou computação simbólica construídas através de sistemas de álgebra computacional. Os softwares mais conhecidos neste campo são os comerciais Mathematica e Maple. Atualmente, porém, cresce o número de softwares e aplicativos livres, que podem ser obtidos gratuitamente. Para computação simbólica pode-se citar o Maxima. O que até poucos anos atrás era prerrogativa do sistema operacional Linux, hoje, muitos dos softwares livres podem ser utilizados em outros sistemas operacionais, como o Windows e o macOS. Assim, o Maxima foi utilizado para resolução de problemas de Equilíbrio Químico. O domínio dessa ferramenta pode ser encarado como a criação de um importante subsunçor ao aluno, que poderá utilizá-lo no aprendizado de outros conceitos.

Para a manipulação de dados, gráficos e ajustes de curvas, uma solução introdutória de grande poder didático são as planilhas eletrônicas. Para esse recurso também são encontradas 
soluções por meio de softwares livres. Utilizou-se o LibreOffice, que oferece uma vasta solução em termos de utilitários de escritório, como editores de texto, planilhas eletrônicas, apresentações, entre outros, que podem ser utilizados em todos os sistemas operacionais mencionados acima.

\section{APLICAÇÃO E DISCUSSÃO}

Nesta seção, além de se ilustrar o resultado da aplicação da sequência descrita, discute-se qualitativamente a participação dos alunos, bem como a percepção do docente a respeito do processo ensino-aprendizagem.

\section{Equilíbrio Químico}

\section{Etapa 1}

Em um primeiro momento, a pergunta-organizador prévio foi: "O que é equilíbrio?". Com essa pergunta, buscou-se, em exercício dialético, a noção de equilíbrio em sua forma geral. Tentou-se induzir ao máximo a participação dos alunos, para darem exemplos e/ou fazerem comentários.

No caso do estudo de Equilíbrio Químico, o encontro na disciplina de QG se iniciou com uma questão problematizadora geral: “O que vocês entendem por Equilíbrio Químico?". Na discussão que se seguiu foi observado que os alunos que se manifestaram já buscavam dar respostas ligadas ao conceito geral de equilíbrio, o que sugere que a atividade com a perguntaorganizador prévio foi bem sucedida. Em seguida, trabalhou-se com um exemplo concreto, do livro-texto (RUSSEL, 1994), que trata do equilíbrio da reação:

$$
2 \mathrm{NOCl}_{(\mathrm{g})}=2 \mathrm{NO}_{(\mathrm{g})}+\mathrm{Cl}_{2(\mathrm{~g})}
$$

A questão busca a concentração $x$ do $\mathrm{Cl}_{2}$. Com os dados, chegou-se à seguinte equação para a constante de equilíbrio: 


$$
K_{c}=\frac{(2 x)^{2}(x)}{(0,25-2 x)^{2}}
$$

Com o valor de $\mathrm{K}_{\mathrm{c}}$ fornecido, faz-se a aproximação de que $\mathrm{x}$ deve ser um número muito pequeno $(x<1)$, de modo que $2 x$ é desprezível diante de 0,25 , e a equação (2) pode ser escrita como:

$$
2 \cdot 10^{-10}=\frac{4 x^{3}}{(0,25)^{2}}
$$

A equação (3) resulta em $x=1,4610^{-4} \mathrm{mols}^{-L^{-1}}$, que, de fato, é muito menor que 0,25 , justificando, assim, a aproximação feita. Com esse valor calculado, encontra-se as concentrações de todas as espécies no equilíbrio. Chega-se com isso ao fim do primeiro encontro de QG (Etapa 1). Para a Etapa 2, é preciso deixar uma questão, uma situação a ser examinada no encontro de IC. A pergunta proposta foi: "e se não pudéssemos fazer a aproximação, como resolver este problema?".

\section{Etapa 2}

O próximo passo é o encontro de IC, que inicia exatamente desse ponto. Uma aula expositiva dialogada inicia com a questão problematizadora deixada na Etapa 1. Perguntou-se: "O quão boa é a aproximação feita, ou seja, $x<<1$ ?". Pela discussão já realizada no encontro de QG, muitos alunos argumentaram se tratar de uma boa aproximação, pois a concentração dos produtos realmente foi bem pequena ante a concentração do reagente no equilíbrio. A contra-argumentação vai na linha do resultado posterior, com a colocação da seguinte questão: "E se não fizéssemos a aproximação, o resultado continuaria pequeno?". Nesse ponto, em socialização de opiniões, chega-se à conclusão que se deve resolver a equação sem aproximação e comparar os resultados. A equação resultante da equação (2) sem aproximação é dada por: 


$$
4 x^{3}-2 \cdot 10^{-10} x^{2}+10^{-10} x-0,125 \cdot 10^{-10}=0
$$

Importante notar que aqui volta-se ao problema do primeiro encontro, com outra perspectiva. Os alunos estão trabalhando sob outro ponto de vista, e isso oportuniza a diferenciação progressiva. Para solução desta questão foram apresentados o software Maxima e o seu módulo de solução de equações.

A Figura 1 mostra a tela do Maxima com a solução da questão. As linhas de entrada (input) e saída (output) são identificadas pelas marcas \%iN e \%ON, respectivamente, que indicam "entrada N" e "saída N". Na primeira linha (\%i3) se encontra a equação aproximada resolvida na aula de QG dentro do comando de solução de equações. Na linha (\%O3) está o resultado sublinhado equivalente ao calculado na aula, porém com mais casas decimais. O Maxima mostra também as duas outras raízes, que são números imaginários, sem significado para o problema real. Notou-se nesse ponto que os alunos mostraram surpresa e ficaram bem impressionados com a obtenção do mesmo resultado calculado em sala mais as raízes imaginárias. Na linha (\%i6) é mostrada a equação não simplificada e na linha (\%06) as raízes obtidas. O valor real sublinhado diverge do primeiro resultado só na terceira casa decimal e é idêntico ao caso aproximado ao se tomar duas casas decimais para respostas. Nesse ponto foi possível discutir a validade da aproximação e a sua interpretação. Notou-se que os alunos trabalharam mais focados no laboratório de informática, imbuídos em encontrar a resposta da situação-problema, e ficaram mais seguros com a aproximação feita.

Figura 1 - Tela do software Maxima com a solução das equações de terceiro grau, com aproximação (linha \%i3) e sem aproximação (linha \%i6)

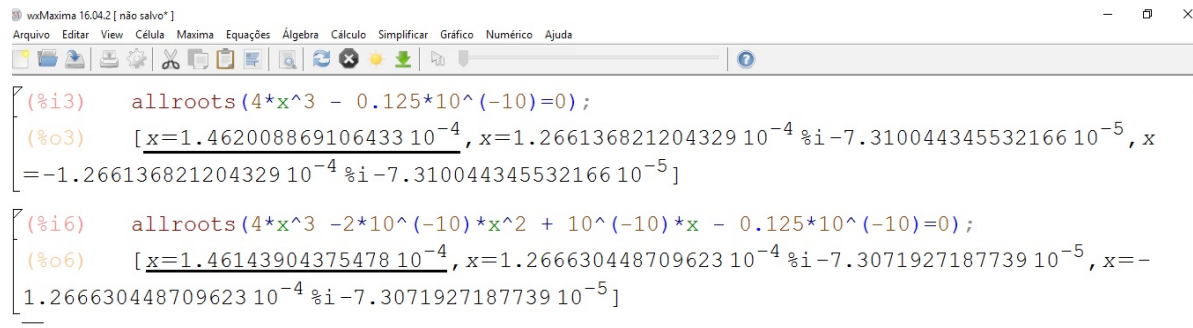

Fonte: elaborada pelos autores, 2017. 


\section{Etapa 3}

No terceiro encontro, na aula de Química Geral, foi feita uma sumarização do conteúdo de Equilíbrio Químico, e novas situações foram discutidas e abordadas com o ferramental matemático desenvolvido. Nesta etapa, observamos que os alunos participaram de forma muito mais efetiva do que se observa na abordagem tradicional, em que a passividade é comum.

\section{Cinética Química}

Para o conteúdo de Cinética Química, utilizou-se a construção de gráficos em planilha eletrônica, com linearização da Equação de Arrhenius. Esse conteúdo foi desenvolvido em 5 encontros (3 de QG e 2 de IC). Os alunos aprenderam a fazer ajustes de curvas e puderam resolver problemas de QG pela planilha nas aulas de IC. Nesse ponto, os alunos puderam explorar os conceitos através dos exercícios práticos, potencializando a diferenciação progressiva e a reconciliação integradora.

\section{COMPARAÇÃO QUANTITATIVA DE DESEMPENHO EM AVALIAÇÕES}

Avaliou-se o desempenho dos estudantes nas questões de prova referentes à Cinética Química nos dois semestres anteriores à aplicação da sequência, comparando-o com o resultado obtido pelos alunos que tiveram a sequência aplicada. Considerando a nota máxima na questão de Cinética Química, nos dois semestres anteriores $12,18 \%$ dos alunos a atingiram, enquanto $15,15 \%$ dos alunos tiveram acerto máximo no semestre em que a sequência foi aplicada pela primeira vez. O resultado mais impactante se deu na faixa de alunos que obtiveram nota igual ou maior a 1,0 (metade do valor máximo): $28,85 \%$ dos alunos apresentaram nota nessa faixa nos dois semestres anteriores. Observou-se um salto para $54,55 \%$ no semestre em que a sequência foi aplicada, quase dobrando o número de alunos com aproveitamento igual ou superior à metade do valor máximo da questão. Para aproveitamento abaixo da metade do valor, passou-se de $71,15 \%$ nos dois semestres anteriores para $45,45 \%$ após a aplicação da sequência. Os dados avaliados mostram uma melhora significativa no desempenho dos alunos nas questões de prova que abordaram o conteúdo estudado com a sequência didática. 


\section{CONSIDERAÇÕES FINAIS}

Neste trabalho se apresentou um relato de experiência da construção e da aplicação de uma sequência didática integrada com base na Teoria de Aprendizagem Significativa, explorando a interdisciplinaridade nas disciplinas de QG e IC para alunos ingressantes nos cursos de Bacharelado e Licenciatura em Química.

Pelo relato de construção, aplicação e resultados dos alunos, acredita-se que a sequência aporta um potencial para a aprendizagem significativa, pois: i) engaja o aluno no processo de aprendizagem, conforme foi observado na aplicação da sequência (aumentando potencialmente a predisposição ao ensino); ii) explora conhecimentos prévios dos alunos tanto nas aulas expositivas dialogadas de QG quanto nas aulas práticas de IC, conforme planejamento; iii) possibilita, conforme planejamento, que a relação do novo conteúdo com o conhecimento prévio seja não arbitrária (as perguntas que funcionam como organizadores contribuem para isso, bem como a retomada de conceitos de Química nas aulas de Computação) e não literal (as aulas expositivas dialogadas possibilitam que os alunos deem significados a essa interação, diferentemente do que ocorre nas aulas expositivas tradicionais); iv) permite, de acordo com a proposta, que os alunos abordem situações de diferentes pontos de vista ("ir" e "vir" no conteúdo) potencializando a diferenciação progressiva e a reconciliação integradora. Os alunos que estudaram conteúdos através da sequência apresentaram um melhor desempenho nas questões de prova relacionadas a tais assuntos. Tudo isso aponta para aspectos muito positivos da proposta e mostra que a aplicação dessa sequência didática deve ser testada com outros conteúdos, buscando um estudo a longo prazo que também viabilize aprimoramentos. Acredita-se que a proposta de sequência, associada a boas escolhas de recursos computacionais e de perguntas usadas como organizadores prévios, pode ser vista como um material potencialmente significativo.

\section{REFERÊNCIAS}

AUSUBEL, David P. Educational psychology - a cognitive view. New York: Holt, Rinehart and Winston, 1968.

AUSUBEL, David P.; NOVAK, Joseph D.; HANESIAN, Helen. Educational psychology: a cognitive view. 2. ed. New York: Holt, Rinehart and Winston, 1978. 
BOGISCH, Maria Inês P.; ALCANTARA, Paulo Roberto. Uma comparação entre estratégias de ensino da Química na educação superior. Revista Diálogo Educacional, v. 3, p. 95-104, 2002.

BRASIL. Ministério da Educação. Secretaria de Educação Média e Tecnológica. Parâmetros Curriculares Nacionais: Ensino Médio. Brasília: Ministério da Educação, 2002.

CARLOS, Jairo G. Interdisciplinaridade no ensino médio: desafios e potencialidades. 2007. 171 f. Dissertação (Mestrado em Ensino de Ciências) - Universidade de Brasília, Brasília, 2007.

FAZENDA, Ivani Catarina A. Interdisciplinaridade: história, teoria e pesquisa. 4. ed. Campinas: Papirus, 1994.

LEITE, Bruno S. Tecnologias no Ensino de Química: Teoria e Prática na Formação Docente. Curitiba: Editora Appris, 2015.

LIMA, Érika R. P. O.; MOITA, Filomena Maria G. da S. C. A tecnologia e o ensino de Química: jogos digitais como interface metodológica. Campina Grande: EDUEPB, 2011.

MENDONÇA, Leda G.; LEITE, Sidnei Q. M.; RODRIGUES, Lúcia R. Aprendizagem significativa nas aulas da graduação tecnológica de Química: Análise da prática pedagógica mediada por teatro. In: ENCONTRO NACIONAL DE PESQUISA EM EDUCAÇÃO EM CIÊNCIAS, 7., 2009, Florianópolis. Anais... Florianópolis: ENPEC, 2009.

MOREIRA, Marco Antônio. Unidades de enseñanza potencialmente significativas - UEPS. Aprendizagem Significativa em Revista/Meaningful Learning Review, v. 1, p. 43-63, 2011.

MOREIRA, Marco Antônio. Aprendizagem significativa: a teoria e textos complementares. São Paulo: Livraria da Física, 2012.

PAIVA, João; RAMOS, Isabel. Adivinhas: Quem é Quem na Tabela Periódica. Química e Ensino, v. 92, p. 61-63, 2004.

PEIXOTO, Joana; ARAÚJO, Cláudia H. S. Tecnologia e educação: algumas considerações sobre o discurso pedagógico contemporâneo. Educação \& Sociedade, v. 33, p. 253-268, 2012.

PINHEIRO, lânderson R. et al. Símbolos químicos com uso da planilha eletrônica do Microsoft Excel. In: ENCONTRO NACIONAL DE PESQUISA EM EDUCAÇÃO EM CIÊNCIAS, 54., 2014, Rio Grande do Norte. Anais... Rio Grande do Norte: CBQ, 2014. 
PONTE, Cristina. Uma geração digital? A influência familiar na experiência mediática de adolescentes. Sociologia, problemas e práticas, v. 65, p. 31-50, 2011.

RUSSELL, John B. Química Geral. São Paulo: Makron Books, 1994. v. 2.

SANDES, Rafael D. D.; AMBROSIO, Renato C.; ANGELUCCI, Camilo A. Integração numérica de leis de velocidade diferenciais com o uso do SCILAB. Química Nova, v. 36, p. 181-186, 2013.

SERRA, Gladis M. D. Contribuição das TIC no ensino e aprendizagem de Ciências: tendências e desafios. 2009. 383 f. Dissertação (Mestrado em Educação) - Universidade de São Paulo, São Paulo, 2009.

TAVARES, Ricarte; SOUZA, Rodolpho O. O.; CORREIA, Alayne O. Um estudo sobre a TIC e o ensino da Química. In: SIMPÓSIO INTERNACIONAL DE INOVAÇÃO TECNOLÓGICA, 1., 2013, Sergipe. Anais... Sergipe: UFS, 2013. p. 657-669.

TERUYA, Leila C. et al. Visualização no ensino de Química: apontamentos para a pesquisa e desenvolvimento de recursos educacionais. Química Nova, v. 36, p. 561-569, 2013.

\section{Julliane D. Yoneda}

Engenheira Química, licenciada e doutora em Química, é professora do Departamento de Química do Instituto de Ciências Exatas da UFF.

jullianeyoneda@id.uff.br

\section{José Augusto Oliveira Huguenin}

Doutor, bacharel e licenciado em Física, é professor do Departamento de Física do Instituto de Ciências Exatas da UFF. É credenciado no Programa de Pós-Graduação em Física da UFF e no Mestrado Nacional Profissional em Ensino de Física, polo 15, UFF/Instituto Federal do Rio de Janeiro.

jose_huguenin@id.uff.br 